\title{
Recoil-induced effects in absorptive optical bistability
}

\author{
R. Bonifacio, ${ }^{1}$ B. W. J. McNeil, ${ }^{2}$ N. Piovella, ${ }^{1}$ and G. R. M. Robb ${ }^{2}$ \\ ${ }^{1}$ Dipartimento di Fisica, Università Degli Studi di Milano, INFN and INFM, Via Celoria 16, Milano I-20133, Italy \\ ${ }^{2}$ Department of Physics and Applied Physics, University of Strathclyde, Glasgow G4 ONG, Scotland
}

(Received 29 March 1999; published 7 January 2000)

\begin{abstract}
We present an analysis of absorptive optical bistability in a system of cold atoms enclosed in a bidirectional ring cavity. This analysis is carried out using a system of equations that extends the so-called Maxwell-Bloch model to self-consistently include atomic center-of-mass motion. The analysis predicts an instability resulting in the simultaneous generation of an atomic density grating and a coherently backscattered radiation field. The formation of the density grating arises from a modulation of the radiation pressure forces exerted on the atoms. A consequence of this instability is that the lower transmission branch of the usual bistability curve, where atomic motion is neglected, can become unstable and the system switches to the upper transmission branch destroying the bistability.
\end{abstract}

PACS number(s): 42.65.Pc, 32.80.Lg, 42.50.Vk

\section{INTRODUCTION}

An optical system which exhibits two steady transmission states for the same input intensity is said to be optically bistable. In this paper we investigate the mean-field theory of optical bistability (OB) of an absorbing system of two-level atoms enclosed in a bidirectional ring cavity. In contrast to previous analyses of absorptive $\mathrm{OB}[1,2]$ we include the atomic center-of-mass motion, which is allowed to evolve in a self-consistent way under the action of the counterpropagating radiation fields within the cavity.

To the authors' knowledge, previous works have only approximated the effects of atomic motion through an inhomogeneous broadening of the atomic transition [3]. Such thermal effects do not require a detailed description of the atomic positions and momenta.

In this paper we consider a sample of initially cold atoms. It is shown that when the center-of-mass dynamics of the atoms are described self-consistently, the system is unstable. This instability simultaneously and spontaneously generates an atomic density grating and a radiation field coherently backscattered by it from the incident pump field. The formation of the density grating is induced by a modulated potential resulting from the interference of the scattered and incident fields. The modulation is a consequence of the nonlinear absorption of the atoms in the standing wave produced by the counterpropagating radiation fields which induces a population difference grating with a period of half the radiation wavelength. The effects of the additional backscattered field and the density grating are shown to have a significant influence on the bistable properties of the ring cavity system.

\section{MODEL}

A schematic of the ring cavity system considered here is shown in Fig. 1. We now outline the derivation of the set of equations used to describe the atom-field interaction within the cavity. These equations extend the so-called MaxwellBloch model [4] to self-consistently include the atomic center-of-mass motion. These equations describe a wide range of radiation-atom interactions, e.g., they have been used to describe a self-cooling mechanism in a strongly driven atomic system [5].

We define the counterpropagating pump and probe radiation electric fields as $\mathbf{E}(z, t)=\left[\mathcal{E}_{1}(z, t) e^{i\left(k_{1} z-\omega_{1} t\right)}\right.$ $+\mathcal{E}_{2}(z, t) e^{-i\left(k_{2} z+\omega_{2} t\right)}+$ c.c. $] \hat{\mathbf{e}}$, where $\hat{\mathbf{e}}$ is a unit vector transverse to the propagation axis $\hat{\mathbf{z}}$ and subscripts 1,2 refer to the probe and pump, respectively. The evolution of the density matrix elements $\rho_{m n},(m, n=1 \cdots 2)$ describes the internal evolution of each atom. The off-diagonal elements $\left(\rho_{21}\right.$ $=\rho_{12}^{*}$ ) describe the polarization as induced by the pump and the probe fields. The dipole moment is then given by $\mathbf{d}$ $=\mu\left(\rho_{12}+\rho_{12}^{*}\right) \hat{\mathbf{e}}$ where $\mu$ is the dipole matrix element. The off-diagonal elements of the density matrix may be written conveniently as a sum of two polarization waves, i.e., $\rho_{12}$ $=S_{1}(z, t) e^{i\left(k_{1} z-\omega_{1} t\right)}+S_{2}(z, t) e^{-i\left(k_{2} z+\omega_{2} t\right)}$. We define $D$ as half the population difference between the lower (1) and upper (2) states, so that $D=\left(\rho_{11}-\rho_{22}\right) / 2$ and $D^{e q}$ is the equilibrium value in the absence of the fields. Here we set $D^{e q}$ $=0.5$ so that the atoms relax to their ground state.

Use of the definitions above in the Bloch equations describing the two-level atomic system, the equation for the force on the $j$ th atom, $F_{z_{j}}=\left.(\mathbf{d} \cdot \partial \mathbf{E} / \partial z)\right|_{z=z_{j}}$, and the Maxwell wave equation yield the following set of coupled scaled differential equations [5]:

$$
\frac{d \widetilde{S}_{1_{j}}}{d \bar{t}}=\left[-\Gamma_{\perp}+i\left(\Delta-\frac{p_{j}}{2}\right)\right] \widetilde{S}_{1_{j}}-2 \rho \widetilde{A}_{1} D_{j}
$$

$$
\begin{aligned}
\frac{d S_{2_{j}}}{d \bar{t}}=\left[-\Gamma_{\perp}+i\left(\Delta+\frac{p_{j}}{2}\right)\right] S_{2_{j}}-2 \rho A_{2} D_{j}, \\
\frac{d D_{j}}{d \bar{t}}=-\Gamma_{\|}\left(D_{j}-D^{e q}\right)+\rho\left[\widetilde{S}_{1_{j}}\left(\widetilde{A}_{1}^{*}+A_{2}^{*} e^{i \theta_{j}}\right)\right. \\
\left.+S_{2_{j}}\left(\widetilde{A}_{1}^{*} e^{-i \theta_{j}}+A_{2}^{*}\right)+\text { c.c. }\right],
\end{aligned}
$$




$$
\begin{aligned}
\frac{d \theta_{j}}{d \bar{t}}=p_{j}, & \\
\frac{d p_{j}}{d \bar{t}}= & -\left(\widetilde{A}_{1} \widetilde{S}_{1_{j}}^{*}-A_{2} S_{2_{j}}^{*}+\widetilde{A}_{1} S_{2 j}^{*} e^{i \theta_{j}}\right. \\
& -A_{2} \widetilde{S}_{1_{j}}^{*} e^{\left.-i \theta_{j}+\text { c.c. }\right),} \\
\frac{d \widetilde{A}_{1}}{d \bar{t}}=\left\langle\widetilde{S}_{1}\right\rangle+\left\langle S_{2} e^{-i \theta}\right\rangle+i \delta \widetilde{A}_{1}-\kappa_{1}\left(\widetilde{A}_{1}-\widetilde{A}_{1}^{\mathrm{eq}}\right), & \\
\frac{d A_{2}}{d \bar{t}}= & \left\langle S_{2}\right\rangle+\left\langle\widetilde{S}_{1} e^{i \theta}\right\rangle-\kappa_{2}\left(A_{2}-A_{2}^{\mathrm{eq}}\right),
\end{aligned}
$$

where the general dependent variables $X \equiv X(\bar{t}), \quad \widetilde{X} \equiv X e^{i \delta \bar{t}}$ and $\quad A_{1,2}=-i \sqrt{2 \epsilon_{0} / n \hbar \omega \rho} \mathcal{E}_{1,2}, \quad \rho=\left(\omega \mu^{2} n / 2 \epsilon_{0} \omega_{r}^{2} \hbar\right)^{1 / 3}$, $p=M\left(\mathrm{v}_{z}-\left\langle\mathrm{v}_{z 0}\right\rangle\right) / \hbar k \rho, \quad \Delta=\left(\omega_{2}+k_{2}\left\langle\mathrm{v}_{z 0}\right\rangle-\omega_{0}\right) / \omega_{r} \rho$, $\delta=\left[2 k\left\langle\mathrm{v}_{z 0}\right\rangle-\left(\omega_{1}-\omega_{2}\right)\right] / \omega_{r} \rho, \quad \bar{t}=\omega_{r} \rho t, \quad \theta=2 k(z$ $\left.-\left\langle\mathrm{v}_{z 0}\right\rangle t\right), \omega_{r}=2 \hbar k^{2} / M$ is the single photon recoil frequency shift, $j=1, \ldots, N,\langle\cdots\rangle=1 / N \sum_{j=1}^{N}(\cdots)$ and we assume, for simplicity, $\Gamma_{\perp}=\Gamma_{\|}=\Gamma=\gamma / \omega_{r} \rho$. In the above scaling, $M$ is the atomic mass, $\gamma$ is the natural linewidth of the transition, $\omega_{0}$ is the transition frequency, $n=n_{s} L_{\mathrm{s}} / L_{\mathrm{cav}}$ is the "reduced" atomic density in the cavity, $n_{s}$ is the atomic density of the sample, $L_{\mathrm{s}}$ is the sample length, $L_{\text {cav }}$ is the cavity length and where appropriate we have assumed $k \approx\left(k_{1}+k_{2}\right) / 2$. Note that for an atomic sample which initially has zero mean velocity $\left(\left\langle\mathrm{v}_{z 0}\right\rangle=0\right), \quad \Delta \rightarrow\left(\omega_{2}\right.$ $\left.-\omega_{0}\right) / \omega_{r} \rho$ and $\delta \rightarrow\left(\omega_{2}-\omega_{1}\right) / \omega_{r} \rho$. Hence $\Delta$ and $\delta$ describe the pump-atom detuning and the pump-probe detuning, respectively, scaled with respect to the "collective recoil bandwidth,' $\omega_{r} \rho[6,7]$. In deriving Eqs. (1)-(7), the pump and probe fields are assumed to be average fields over intervals $\Delta z \sim \lambda$, the radiation wavelength, consistent with the slowly varying envelope approximation (SVEA) [4]. The mean, $\langle\cdots\rangle$, refers to the $N$ atoms within that interval.

We have assumed that the mean field limit can be applied when describing the evolution of both the pump and the probe. Cavity losses are assumed to be equal for both pump and probe, i.e., $\kappa_{1}=\kappa_{2}=\kappa$, where $\kappa=-c \ln (R) / \omega_{r} \rho L_{\text {cav }}$ is the scaled cavity loss rate. Here $\widetilde{A}_{1}^{\mathrm{eq}}$ and $A_{2}^{\mathrm{eq}}$ are the constant

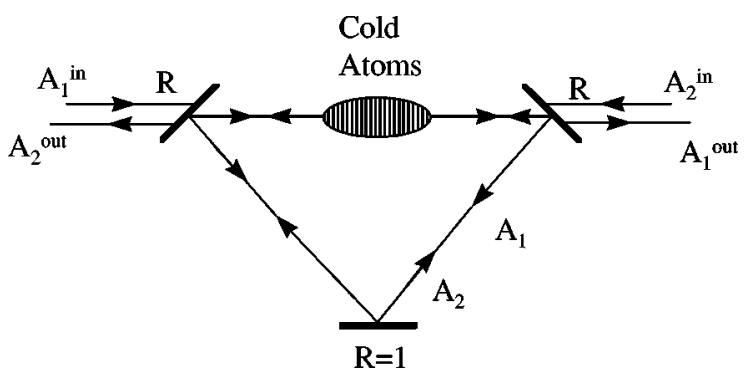

FIG. 1. A schematic diagram showing a cold atomic sample enclosed in a bidirectional ring cavity. Note that $A_{1,2}^{\mathrm{eq}}$ $=A_{1,2}^{\text {in }} / \sqrt{1-R}$ and $A_{1,2}^{\text {out }}=A_{1,2} \sqrt{1-R}$ where $R$ is the reflectivity of the output mirrors.

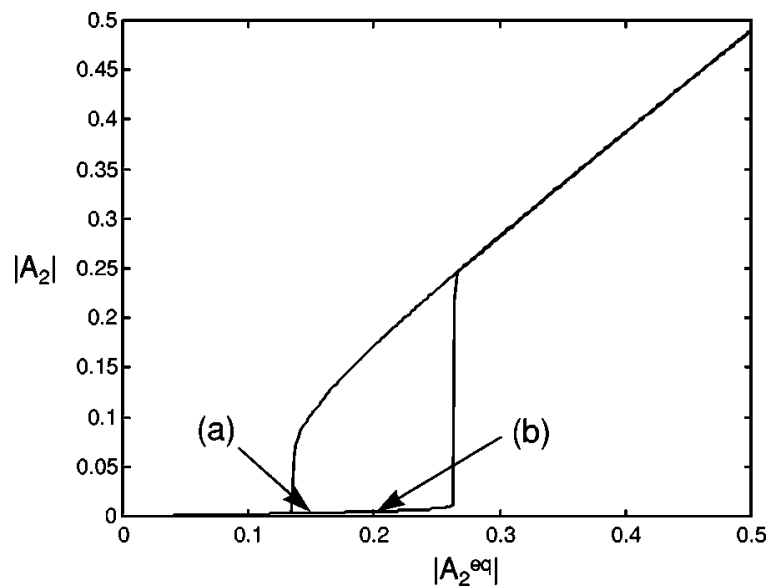

FIG. 2. Pump field amplitude $\left|A_{2}\right|$ in the cavity as a function of the injected pump field amplitude $\left|A_{2}^{\mathrm{eq}}\right|$ for $\rho=1500, \kappa=1, \Gamma$ $=30, \Delta=0$, and $\delta=0$, giving a cooperation parameter $C=25$.

probe and pump fields input to the cavity. It has been assumed that the pump frequency coincides with that of a mode of the cavity, i.e., $\omega_{2}=\omega^{(m)}=2 \pi m c / L_{\text {cav }}$ where $m$ is an integer. Furthermore, momentum diffusion due to spontaneous emission is neglected in this model. This point is discussed in more detail in Sec. V.

\section{ABSORPTIVE OPTICAL BISTABILITY}

We now use this model to demonstrate the well-known phenomenon of absorptive optical bistability in the absence of atomic center-of-mass motion and for a unidirectional ring cavity. To do this, we set $d \theta / d \bar{t}=d p / d \bar{t}=\widetilde{A}_{1}=\widetilde{S}_{1}=0$. It can be shown that the resulting Eqs. (2), (3), and (7) have bistable solutions when the condition $C>4$ is satisfied, where $C=\rho / 2 \kappa \Gamma$ is the cooperation parameter [3]. Optical bistability is demonstrated in the usual way in Fig. 2 by plotting the scaled field amplitude $\left|A_{2}\right|$ as a function of the scaled input field amplitude $\left|A_{2}^{\text {eq }}\right|$ for $C=25$. We will use this example to study the stability of the solutions when atomic motion is included in the model.

\section{RECOIL-INDUCED INSTABILITY}

The restrictions in Sec. III are now relaxed and we consider the general case of a bidirectional ring cavity including atomic center-of-mass motion. We investigate the stability of the solutions for $A_{2}$ on the lower branch of the bistability curve of Fig. 2 for small initial values of $A_{1}$, simulating a noise field, and a cold atomic sample. An equilibrium value $A_{2}^{\text {eq }}$ and initial value of $A_{2}$ close to the steady-state solution for $A_{2}$ is taken from the lower branch of Fig. 2. The system of Eqs. (1)-(7) is then integrated.

The behavior of the system is investigated for different values of $A_{2}^{\mathrm{eq}}$ and $A_{2}$ corresponding to points lying close to the lower branch of Fig. 2 when $A_{1}^{\mathrm{eq}}=0$ and $A_{1_{0}}=10^{-3}$.

The solution of Eqs. (1)-(7) for point (a) is shown in Fig. 3. Figure 3 shows the evolution of $\left|A_{2}\right|^{2},\left|A_{1}\right|^{2}$, and $|b|$ for conditions corresponding to point (a) on Fig. 2. It can be 


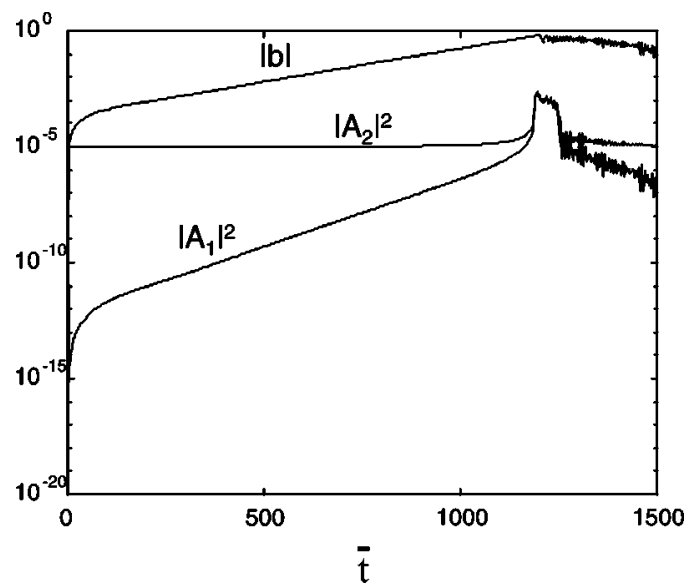

FIG. 3. Evolution of the probe intensity $\left|A_{1}\right|^{2}$, pump intensity $\left|A_{2}\right|^{2}$, and bunching parameter amplitude $|b|$ in the cavity as a function of scaled time $\bar{t}$ for $A_{2}^{\mathrm{eq}}=0.15, \rho=1500, \kappa=1, \Gamma$ $=30, \Delta=0$, and $\delta=0$.

seen that $\left|A_{1}\right|$ and $|b|$ display exponential growth, but when $\left|A_{1}\right| \approx\left|A_{2}\right|$ the value of $\left|A_{2}\right|$ undergoes a sudden transition to a larger value before returning to its approximately constant value which is very close to that when atomic motion is neglected. The bunching parameter $b=\left\langle e^{-i \theta}\right\rangle$ is a measure of the spatial distribution of the atoms on the wavelength scale. The behavior of $|b|$ shown in Fig. 3 indicates that the initially uniformly distributed atoms become strongly bunched on a scale of $\lambda / 2$. A physical mechanism for this bunching is described in Sec. V.

Figure 4 shows the evolution of $\left|A_{1}\right|^{2}$ and $\left|A_{2}\right|^{2}$ and the bunching parameter $|b|$ for conditions corresponding to point (b) on Fig. 2. In this case, when $\left|A_{1}\right|$ grows to become approximately equal to $\left|A_{2}\right|,\left|A_{2}\right|$ undergoes a sudden transition to a higher steady state value. This steady state for $\left|A_{2}\right|$ is approximately equal to the upper branch of the bistable curve on Fig. 2. Therefore it has been shown that for a suf-

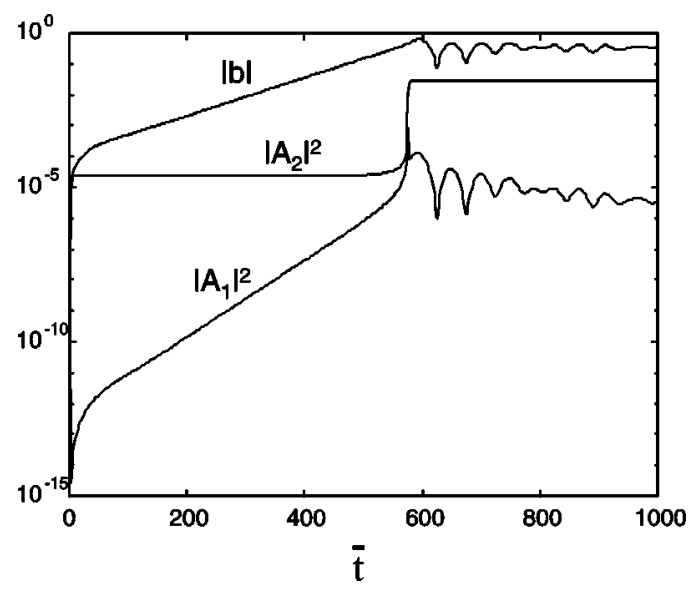

FIG. 4. Evolution of the probe intensity $\left|A_{1}\right|^{2}$, pump intensity $\left|A_{2}\right|^{2}$, and bunching parameter amplitude $|b|$ in the cavity as a function of scaled time $\bar{t}$ for $A_{2}^{\mathrm{eq}}=0.2, \rho=1500, \kappa=1, \Gamma$ $=30, \Delta=0$, and $\delta=0$. Switching of the pump to the high transmission state occurs at $\bar{t} \approx 550$. ficiently large value of $\left|A_{2}^{\mathrm{eq}}\right|$, the lower branch of the bistability curve for "fixed" atoms in a unidirectional ring cavity becomes unstable when an additional counter-propagating wave is introduced and the atomic center-of-mass motion is included. Note that this counter-propagating wave can arise from noise in the system. That this instability is intimately linked to atomic motion and not just due to induced effects in stationary atoms can be shown by setting $d \theta / d \bar{t}=d p d \bar{t}$ $=0$ in Eqs. (1) $-(7)$ while letting $S_{1}$ and $A_{1}$ evolve. When this is carried out, $\left|A_{1}\right|$ does not deviate from its initially small value and $\left|A_{2}\right|$ attains a steady-state value equal to that when atomic motion is neglected and the evolution of $A_{1}$ is suppressed (Fig. 2).

\section{ANALYSIS}

In this section we show how two counter-propagating fields resonant with an ensemble of two-level atoms can produce a force which acts to spatially bunch the atoms. If we assume for simplicity that the atomic momentum is sufficiently small that $p \ll \Gamma$, and that $\Gamma \gg \kappa$, it is possible to adiabatically eliminate the "internal" atomic variables $S_{1}, S_{2}$, and $D$ for the $j_{\text {th }}$ atom and solve for $D_{j}$. If we assume weak nonlinearity $\left(4 \rho^{2}\left|A_{1,2}\right|^{2} \ll \Gamma^{2}\right)$ then the result is

$$
D_{j} \approx \frac{1}{2}\left[1-\frac{4 \rho^{2}}{\Gamma^{2}} I\left(\theta_{j}\right)\right]
$$

where $I(\theta)=\left|A_{1}\right|^{2}+\left|A_{2}\right|^{2}+A_{1} A_{2}^{*} e^{i \theta}+A_{1}^{*} A_{2} e^{-i \theta}$ is the total scaled intensity due to the interference of the probe and pump fields. For the case where the atoms are uniformly distributed in space, it can be seen from Eq. (8) that the population difference contains a sinusoidal variation due to the modulated intensity $I(\theta)$. We now show that this "population difference grating" can give rise to a bunching force by substituting for $S_{1}, S_{2}$, and $D$ in the force equation (5) using (8), which produces

$$
\frac{d p_{j}}{d \bar{t}}=\frac{2 \rho}{\Gamma}\left(\left|A_{1}\right|^{2}-\left|A_{2}\right|^{2}\right)\left[1-\frac{4 \rho^{2}}{\Gamma^{2}} I\left(\theta_{j}\right)\right] .
$$

The first term in Eq. (9) is the usual radiation pressure force, which is position $(\theta)$ independent. The second term in Eq. (9) describes a reduction in the radiation pressure force due to nonlinear "bleaching" of the atoms' response to the field. Note that this bleaching is position dependent, giving rise to a force which is modulated with the radiation intensity that the atoms experience. It is this modulated force which acts to bunch the atoms in space to form a density grating.

If we now assume that $\left|A_{2}\right|$ is constant and $A_{1}, b$, and $p$ are small quantities, it can be shown from a linear analysis of Eqs. (1)-(6) using the method of collective variables [8] that both $A_{1}, b \propto e^{\lambda t}$ and therefore both will grow exponentially if the cubic

$$
\lambda^{3}+\left(\frac{\rho}{\Gamma}+\kappa\right) \lambda^{2}+i \frac{\Omega^{4}}{2 \Gamma^{4}}\left(\frac{\lambda}{2 \Gamma}-1\right)=0
$$


has any solutions with $\operatorname{Re}(\lambda)>0$. It can be shown that

$$
\operatorname{Re}(\lambda) \approx \frac{\Omega^{2}}{2 \Gamma^{2}} \frac{1}{\sqrt{\left(\kappa+\frac{\rho}{\Gamma}\right)}}
$$

where $\Omega=2 \rho\left|A_{2}\right|$ is the scaled pump Rabi frequency, so $\operatorname{Re}(\lambda)>0$ always. These results are consistent with numerical solutions of the fully nonlinear model.

It was stated in Sec. II that momentum diffusion due to spontaneous emission and the heating of the atoms it causes is neglected in our analysis. We now derive the condition which must be satisfied in order to justify the neglect of momentum diffusion effects. Atoms undergoing spontaneous emission emit photons in random directions. Consequently, the atoms feel randomly directed momentum "kicks" of magnitude $\hbar k$. The long term effect of this is to cause a random walk in momentum space, heating the atomic sample. For simplicity, we consider the effect of spontaneous emission as a one-dimensional random walk. $N$ spontaneous emission events correspond to N momentum "steps" of magnitude $\hbar k$, which gives rise to an atomic momentum spread [9], which in the scaled notation of this paper can be expressed as

$$
\left\langle p^{2}\right\rangle \approx 2 D_{p} \bar{t}
$$

where $D_{p}=\Gamma \rho_{22} / 2 \rho^{2}$ is the scaled diffusion coefficient and $\rho_{22}$ is the probability of finding an atom in the upper energy state.

The effect of a constant Gaussian atomic momentum spread, of width $\sigma=\sqrt{\left\langle p^{2}\right\rangle}$, is to cause the amplitude of the density grating, described by $|b|$, to decay as [7]

$$
|b(\bar{t})| \propto \exp \left(-\frac{\sigma^{2} \bar{t}^{2}}{2}\right) .
$$

An estimate of the effect of a growing Gaussian momentum spread, can be obtained by substituting for $\sigma^{2}$ in (13) using (12), resulting in $|b(\bar{t})| \propto \exp \left(-D_{p} \bar{t}^{3}\right)$. A more rigorous analysis gives the similar result $[10]$ of $|b(\bar{t})|$ $\propto \exp \left(-4 D_{p} \vec{t}^{3} / 9\right)$. In order to neglect the effects of momentum diffusion, the decay of the grating due to the induced atomic momentum spread must be negligible during $\tau_{g}$, the scaled growth time of the density grating due to the instability. The condition for the neglect of momentum diffusion due to spontaneous emission is then $4 D_{p} \tau_{g}^{3} / 9 \ll 1$ or

$$
\frac{\Gamma}{9 \rho^{2}} \frac{\Omega^{2}}{\left(\Gamma^{2}+\Omega^{2}\right)} \tau_{g}^{3} \ll 1,
$$

where the relation $\rho_{22}=\Omega^{2} / 2\left(\Gamma^{2}+\Omega^{2}\right)$ has been used. From Fig. 4 , it can be seen that $\tau_{g} \approx 70$ and $\left|A_{2}\right| \approx 0.0048$, so for the parameters used in Fig. 4, the LHS of (14) is approximately 0.095 . The neglect of momentum diffusion due to spontaneus emission in this example is therefore consistent with the physical arguments described above.

\section{CONCLUSIONS}

We have shown that when describing absorptive optical bistability in a ring cavity containing cold atoms, the atomic center-of-mass motion must be included in the description in a self-consistent way. When this motion is included, the lower branch of the bistability curve neglecting atomic motion can become unstable and the transmission switches to the upper branch, i.e., the system is no longer truly bistable. This behavior results from the simultaneous generation of an atomic density grating and a coherently backscattered radiation field. The formation of the density grating arises from a modulation of the radiation pressure forces exerted on the atoms. This causes the atoms to bunch near the nodes of the standing wave where there is little absorption. The cavity therefore appears "empty" to the pump and switches to the high transmission state, subsequently bleaching the atoms.

It should be noted that density grating formation has also been predicted in a system of cold atoms which have a predominantly dispersive interaction with the radiation field $[6,7,10]$. It could be expected therefore that optical bistability in a system of dispersive cold atoms would be significantly affected by the inclusion of atomic center-of-mass motion in the description of the interaction. Furthermore, in common with normal OB it would be expected that the experimental observation of the effects of atomic recoil on OB could be observed more easily in a dispersive system, as the effects of momentum diffusion due to spontaneous emisson would be much smaller, and the atomic transition would not require to be saturated. A detailed analysis of absorptive and dispersive OB including the effects of atomic recoil and a description of possible experiments, will be presented in a future extended publication [11].

\section{ACKNOWLEDGMENTS}

The authors would like to thank the Royal Society of Edinburgh and the EPSRC for support of G.R.M.R. and B. McN., respectively.
[1] R. Bonifacio and L. Lugiato, Opt. Commun. 19, 172 (1976).

[2] R. Bonifacio and L. Lugiato, Lett. Nuovo Cimento 21, 505 (1978).

[3] L. Lugiato, Prog. Opt. XXI, 69 (1984), and references therein.

[4] F.T. Arecchi and R. Bonifacio, IEEE J. Quantum Electron. 1, 169 (1965).

[5] R. Bonifacio, B.W.J. McNeil, and G.R.M. Robb, Opt. Com- mun. 161, 1 (1999).

[6] R. Bonifacio, L. De Salvo, L.M. Narducci, and E.J. D’Angelo, Phys. Rev. A 50, 1716 (1994).

[7] R. Bonifacio, G.R.M. Robb, and B.W.J. McNeil, Phys. Rev. A 56, 912 (1997), and references therein.

[8] L. Desalvo, R. Cannerozzi, R. Bonifacio, E.J. D'Angelo, and L.M. Narducci, Phys. Rev. A 52, 2342 (1995). 
[9] See, e.g., S. Stenholm, in Proceedings of the International School of Physics “Enrico Fermi,"' Course CXVIII, edited by E. Arimondo, W. D. Phillips, and F. Struma (North-Holland, Amsterdam, 1992), p. 29.
[10] M.G. Moore and P. Meystre, Phys. Rev. A 58, 3248 (1998).

[11] R. Bonifacio, B.W.J. McNeil, N. Piovella, and G.R.M. Robb (unpublished). 